\title{
Extravehicular Activity Micrometeoroid and Orbital Debris Risk Assessment Methodology
}

\author{
Kevin D. Hoffman ${ }^{\mathrm{a}}$, James L. Hyde ${ }^{\mathrm{a}}$, Eric L. Christiansen ${ }^{\mathrm{b}}$, and Dana M. Lear ${ }^{\mathrm{b}}$, \\ ${ }^{a}$ Jacobs, NASA Johnson Space Center, Mail Code XI, Houston, TX 77058, USA \\ ${ }^{b}$ NASA Johnson Space Center, Mail Code XI, Houston, TX 77058, USA
}

\begin{abstract}
A well-known hazard associated with exposure to the space environment is the risk of vehicle failure due to an impact from a micrometeoroid and orbital debris (MMOD) particle. Among the vehicles of importance to NASA is the extravehicular mobility unit (EMU) "spacesuit" used while performing a US extravehicular activity (EVA). An EMU impact is of great concern as a large leak could prevent an astronaut from safely reaching the airlock in time resulting in a loss of life. For this reason, a risk assessment is provided to the EVA office at the Johnson Space Center (JSC) prior to certification of readiness for each US EVA.
\end{abstract}

Keywords: Extravehicular activity; Micormeteoroid; Orbital debris

\section{EVA Assessment Overview}

EVA assessments are performed using standard risk assessment methodology illustrated in Figure 1 (Christiansen, 2003). Central to the process is the Bumper risk assessment code (Figure 2), which calculates the critical penetration risk based on geometry, shielding configurations and flight parameters.

The assessment process begins by building a Finite Element Model (FEM) of the EMU, which defines the size and shape of the EMU as well as the locations of the various shielding configurations. Each region of the EMU having a different shield configuration and/or failure criteria are given a unique property identifier (PID) number in the FEM. The model was built using the NX I-deas software package from Siemens PLM Software. The FEM was constructed using triangular and quadrilateral elements that define the outer shell of the EMU. Bumper-3 uses the model file to determine the geometry of the EMU for the analysis.

The next step of the process is to identify the failure criteria and appropriate ballistic limit characteristics for the various regions of the FEM. The two failure criteria for the EMU are perforation threshold failure and a critical hole size threshold failure of the EMU bladder. Perforation threshold failure would result in any size leak in the EMU bladder while the critical hole size threshold failure ( $>4 \mathrm{~mm}$ hole in the bladder) would result in an uncontrolled leak. The ballistic limit equations define the critical size particle that will cause failure (as defined by the failure criteria) at a given impact angle and impact velocity. When the finite element model is built, each individual element is assigned a PID to act as an index for its shielding properties. Ballistic limit equations are based on results from impact tests on samples that are representative of the vehicle shields. The appropriate ballistic limit equations (BLEs) are added to the Bumper-3 code as needed for each and every PID in the model.

The final stage of the analysis is to determine the predicted number of failures for the EMU. This is calculated using the micrometeoroid and orbital debris (MMOD) environment definitions that are specified by the spacecraft design. The MMOD environment models predict an impacting particle flux based on inputs for orbital parameters, spacecraft attitude and analysis date. 
Using Poisson statistics, and combining the previously defined geometry, shielding characteristics and ballistic limit equations with the MMOD environment model calculations, the Bumper-3 code determines a predicted number of failures for the EMU.

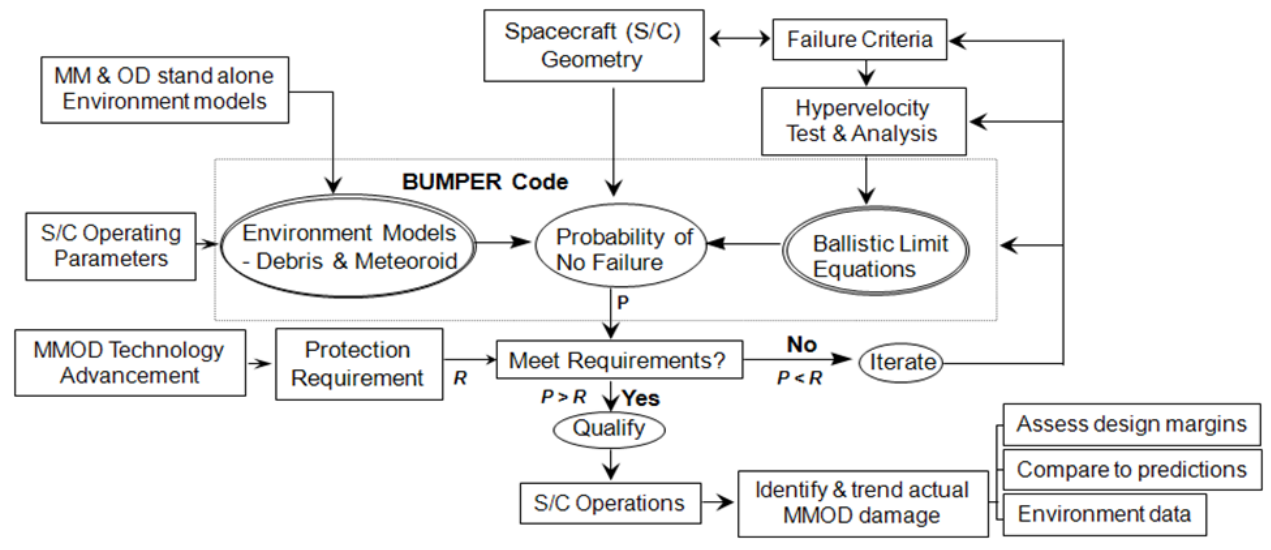

Fig. 1. MMOD Risk Assessment Flowchart

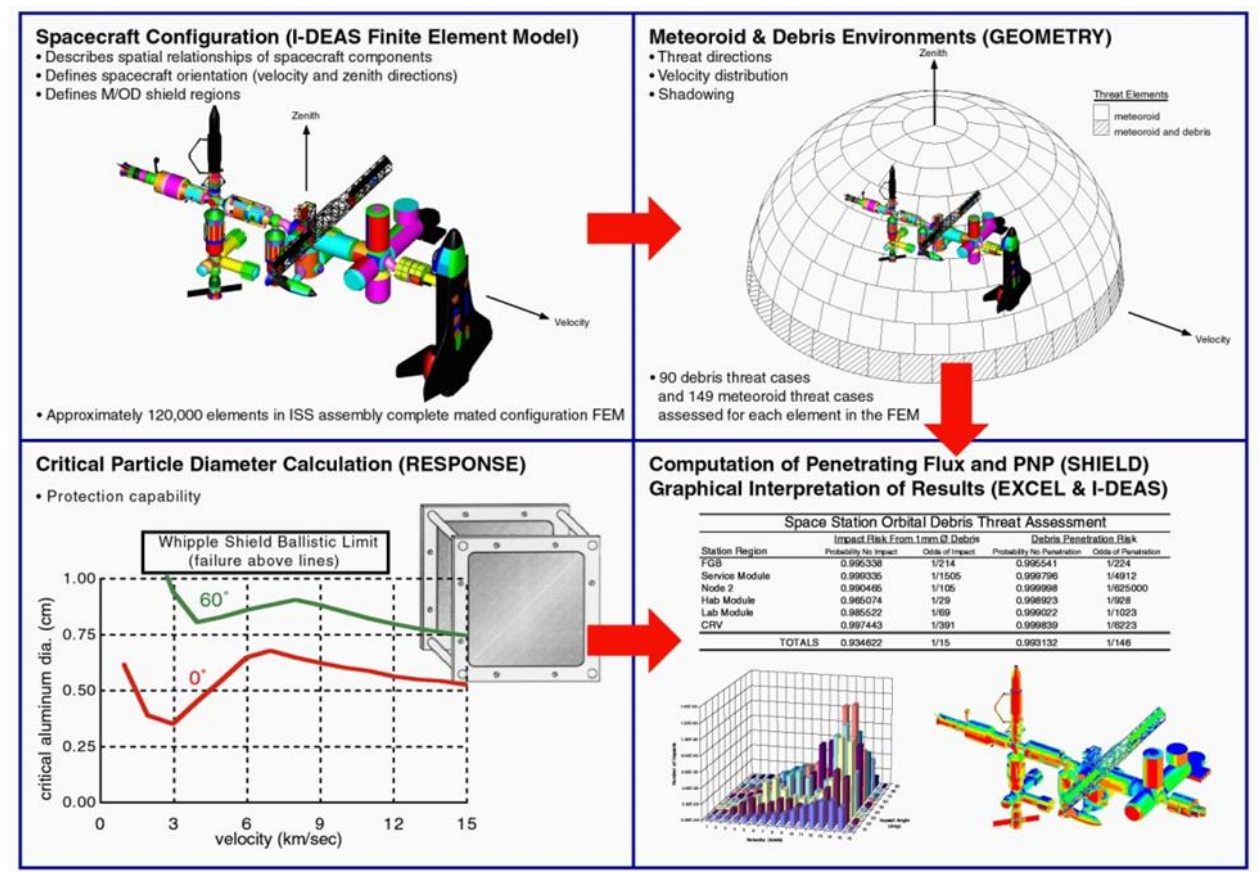

Fig. 2. Bumper-3 MMOD Risk Assessment Code

\subsection{US EVA Timeline Analysis}

For every US EVA, a timeline MMOD risk analysis is provided to the EVA office at JSC prior to the certification of EVA readiness. With assistance from the EVA office, a summary of EMU positions (including body orientation) for the specific EVA worksite locations on the International Space Station (ISS) is produced using detailed EVA summaries/presentations and/or EVA training run videos from the Neutral Buoyancy Lab (NBL.) From this information, the analysis FEMs are built by orienting an 
EMU FEM at each worksite location on a simplified ISS FEM. When one or more worksites require multiple body orientations, additional analysis FEMs are built. The analysis FEM used for the US 52 and 53 EVAs is shown in Figure 3.

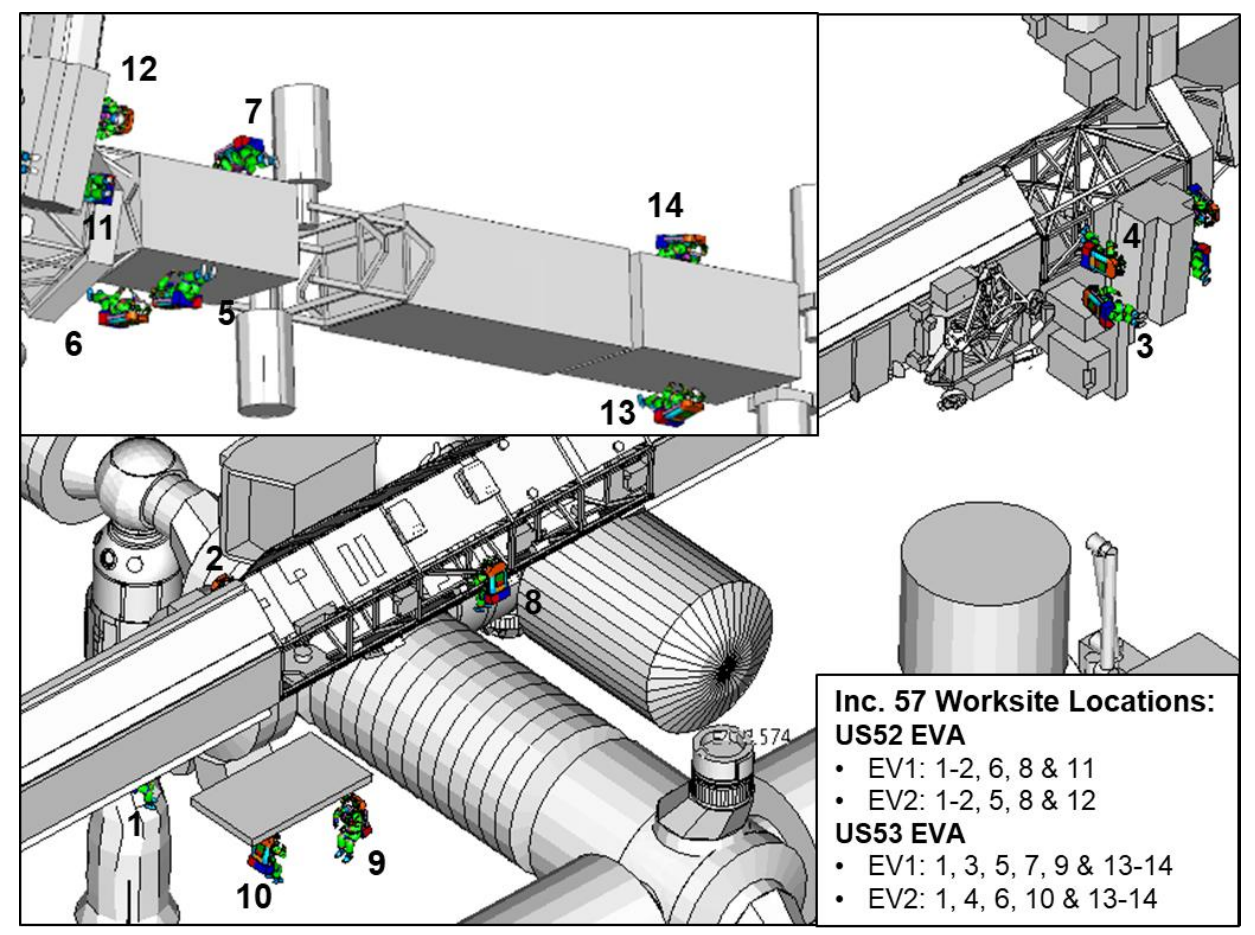

Fig. 3. Increment 57 (US EVAs 52 \& 53) Analysis Model including ISS Shadowing

The analysis FEM(s), environment files and BLE parameters are inputted into the Bumper-3 code to obtain the expected number of failures at each worksite for a one year exposure. These results are scaled based on actual worksite exposure duration to generate total EVA risk and individual EMU risk for two failure criteria (any size leak and catastrophic leak.) Meteoroid and orbital debris enhancement factors (see Section 1.2) are applied to obtain the failure odds for the planned EVA date.

\subsection{Meteoroid and Orbital Debris Enhancement Factors}

The meteoroid and orbital debris environment files used for the timeline analysis provide the average flux for a single year exposure duration. Due to the short exposure duration of 6 to 7 hours, EVAs are more sensitive to sporadic events outside of the single year background environment (i.e. orbital debris breakups and meteor showers.) Environment enhancement factors are used to account for these events. For every US EVA, the Meteoroid Environment Office (MEO) at Marshall Space Flight Center (MSFC) provide 6-hour meteoroid enhancement factors for each day of the EVA window. The fluence factor is for particles with a kinetic energy of at least $6.7 \mathrm{~J}$ (the kinetic energy of a $0.04 \mathrm{~cm}$ diameter meteoroid traveling at $20 \mathrm{~km} / \mathrm{s}$.) For example, meteoroid enhancement factors provided by the MEO for the December 2018 window are shown in figure 4 and indicate a peak fluence enhancement factor of nearly $40 \%$ for an EVA performed during the Geminids meteor shower on December $14^{\text {th }}, 2018$. Likewise, the Orbital Debris Program Office (ODPO) at JSC provide an orbital debris enhancement factor to account for any recent orbital debris breakups that might increase the flux at ISS orbit. 


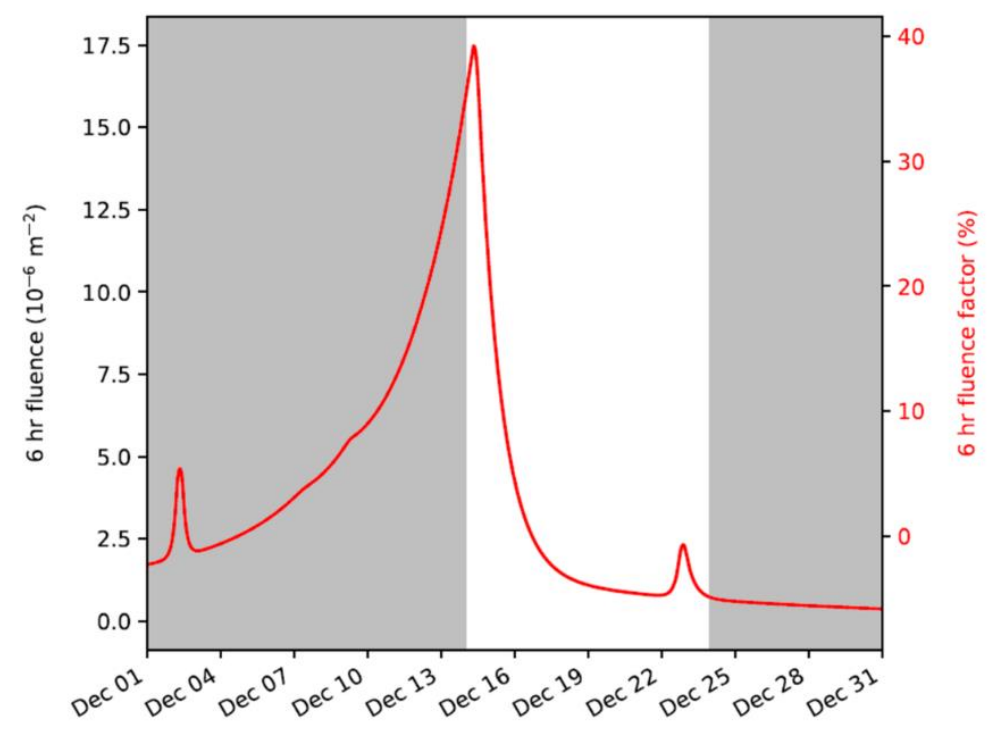

Fig. 4. Increment 57 (US EVAs 52 \& 53) Meteoroid Environment Enhancement Factors

\section{EMU Finite Element Model}

A detailed finite element model (FEM) of the EMU (shown in Figure 5) has been created which has regions for the various shielding configurations. Each shielding configuration is based on the layers and materials over the innermost bladder layer that maintains the acceptable atmospheric environment for the astronaut. The EMU outer mold line is defined by over 14,500 quadrilateral and triangular elements. The color changes in the model views shown in Figure 5 illustrate the 42 different surface property ID (PID) types.
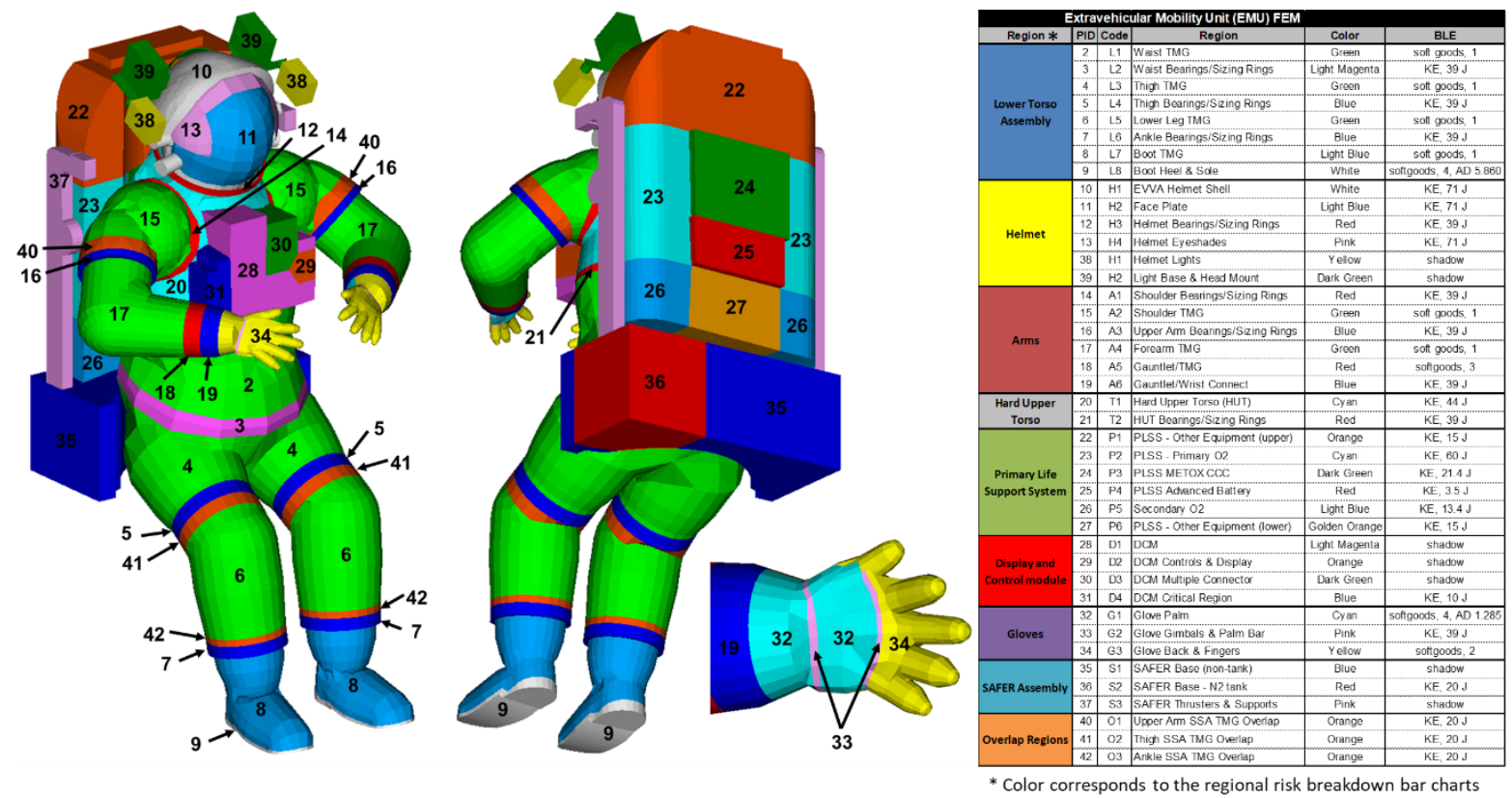

Fig. 5. Extravehicular Mobility Unit (EMU) Finite Element Model (FEM) 


\section{Hypervelocity Impact Testing}

The EMU can be subdivided into two major shielding configurations: soft goods and hard goods. The soft goods (fabric layers over inner bladder) include the arms, legs, lower torso, gloves and boots overlap regions while the hard goods include the helmet, face plate, sizing rings, hard upper torso (HUT), portable life-support system (PLSS), and the display and control module (DCM.) 65 "soft goods" and over 40 "hard goods" hypervelocity impact tests were performed on EMU samples at the White Sands Test Facility. Testing of "soft goods" included both pressurized and unpressurized bladders with no difference found in the ballistic limit with pressurization. Testing results were used to set the ballistic limit equations (BLE) for each shielding configuration.

\section{EMU Random Orientation Risk Study}

A risk assessment of the EMU was performed using the ORDEM 3.0 orbital debris environment and the MEM R2 meteoroid environment. Twenty-four distinct attitude (body orientation) runs of the unshadowed EMU FEM were made for the exposure period of 2018 to 2024. Figure 6 shows the percentage of total risk contribution for the main regions of the EMU, with $75 \%$ of the total risk from the soft goods regions of the lower torso assembly, arms and gloves. The risk to area ratio for each of these sections is shown in Figure 7 and highlights that the gloves carry the highest risk per area for the EMU. Figure 8 is a risk contour (red areas are higher risk, blue areas are lower risk) and indicates the soft good regions of the gloves, arms, legs and torso carry the most risk.

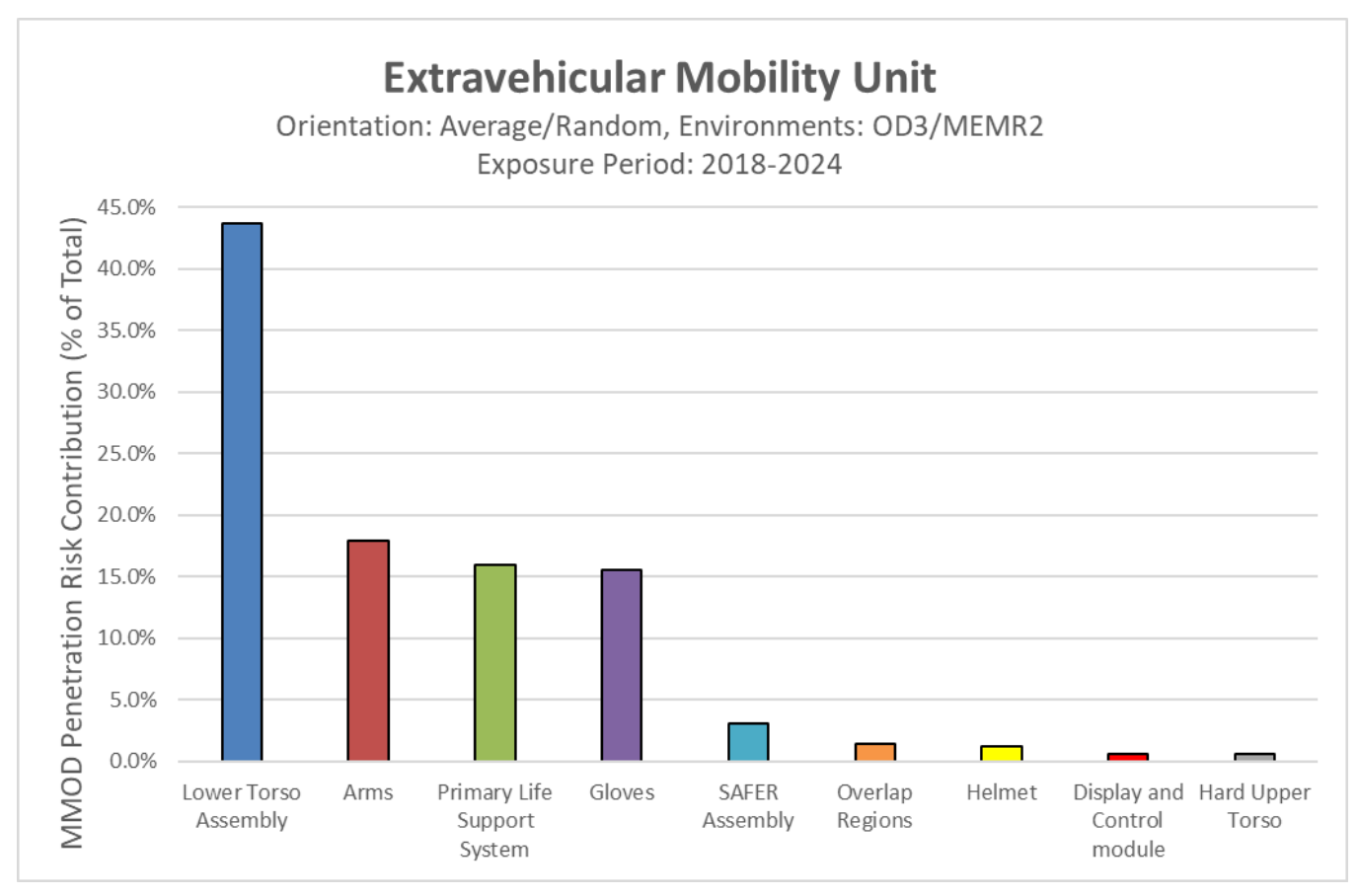

Fig. 6. Extravehicular Mobility Unit (EMU) Unshadowed Penetration Risk Summary (ORDEM3 and MEM R2 environments) 


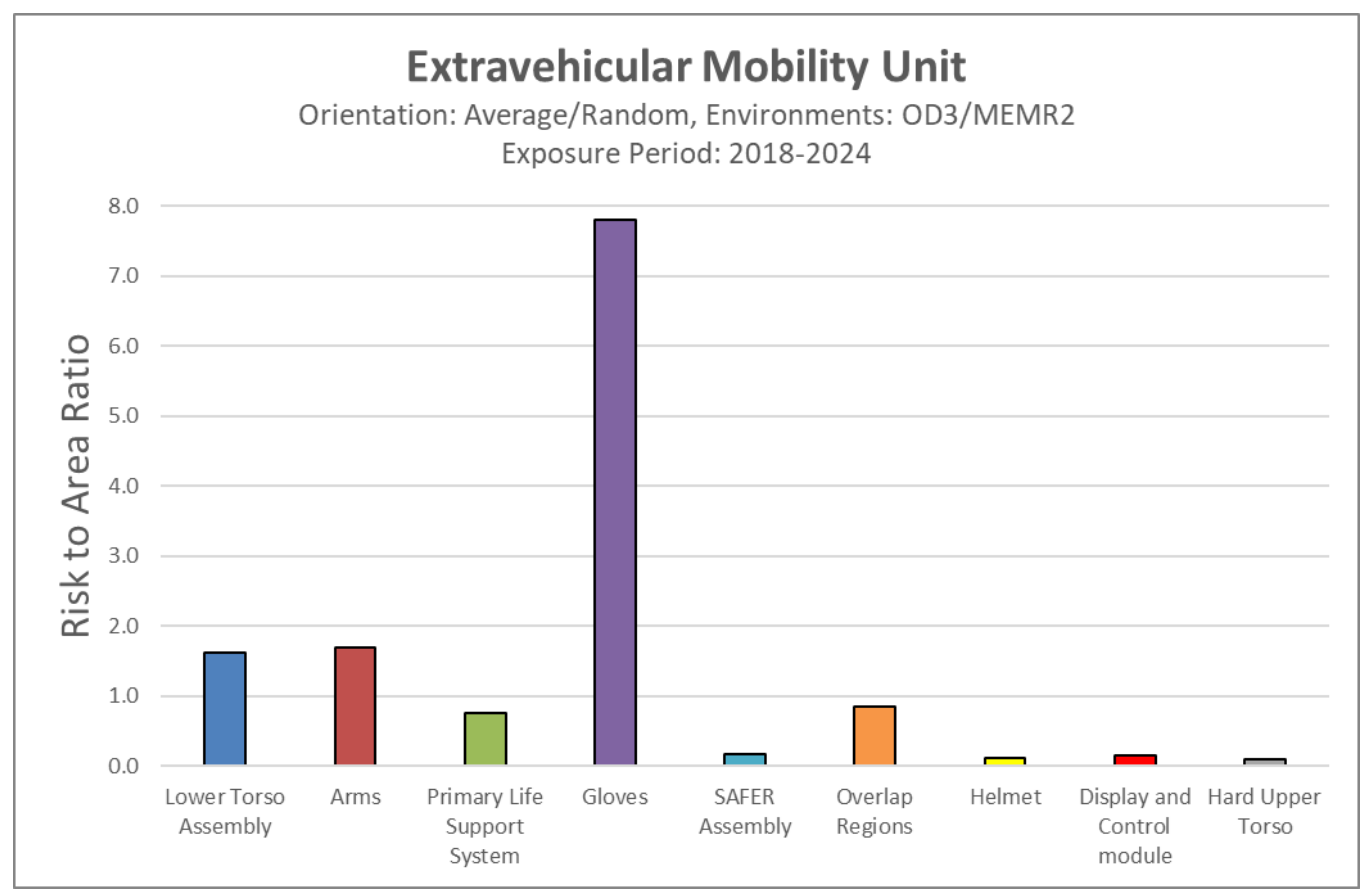

Fig. 7. Extravehicular Mobility Unit (EMU) Unshadowed Penetration Risk to Region Area Summary (ORDEM3 and MEM R2 environments)
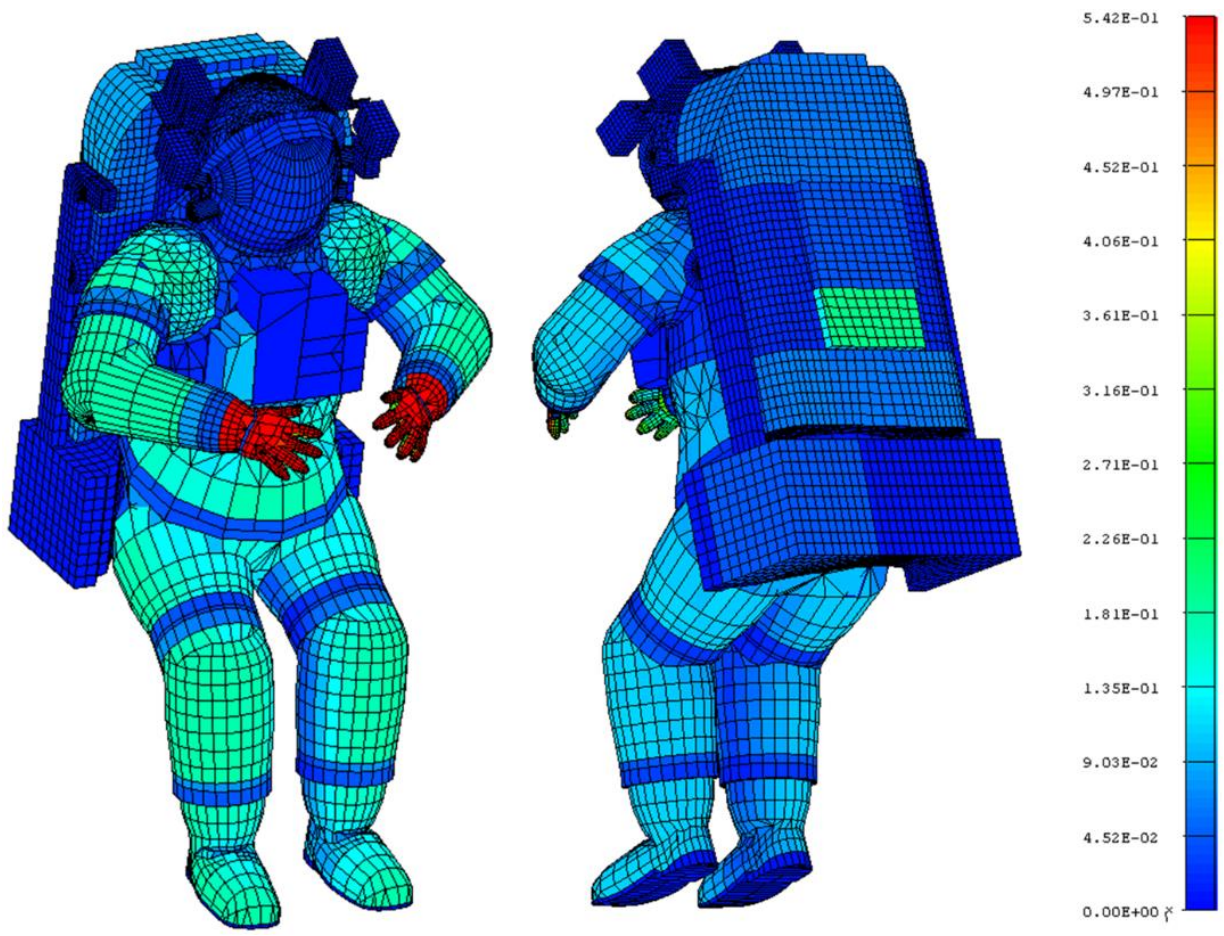

Fig. 8. Extravehicular Mobility Unit (EMU) Unshadowed Average Risk Contour Plot (red areas $=$ higher risk, blue areas $=$ lower risk) 


\section{Acknowledgements}

In support of the Jacobs JSC Engineering, Technology and Science (JETS) contract (NNJ13HA01C) for the Astromaterials Research and Exploration Science (ARES) division at the NASA Johnson Space Center (JSC.)

\section{References}

[1] Christiansen, Eric L., 2003. Meteoroid/Debris Shielding, NASA TP-2003-210788 . Houston: NASA

[2] Friesen, L.J. and Christiansen, E.L., 1997. Hypervelocity Impact Tests of Extravehicular Mobility Unit (EMU) (Space Suit) Material Samples, Part 1, JSC 27856, Houston: NASA

[3] Lyons, Frankel, 1998. Hypervelocity Impact Testing of Extravehicular Mobility Unit (EMU) Alternative Material Samples, Part 1, JSC 28445, Houston: NASA 